\title{
Analysis of Ultrasound Image Using Wavelet And Curvelet Transform
}

\author{
[Sheth Dhrumil H., Shah Saurin R.]
}

\begin{abstract}
Ultrasound images are normally affected by speckle noise which is due to the coherent nature of scattering phenomenon. Speckle filtration is accompanied with loss of diagnostic features. Despeckling techniques are to reduce the speckle noise in Ultrasound images, provided that the structural features and edges. This paper gives detailed information regaurding discriminating power of the various multiresolution based thresholding techniques i.e. Wavelet, curvelet for denoise the image. Curvelet transform offer exact reconstruction, ease of implementation, stability against perturbation, and low computational complexity. Finally these two methods are compared and the performance are verified by quality metric analysis.
\end{abstract}

Keywords-curvelet, wavelet, speckle noise, thresholding, ultrasound image.

\section{Introduction}

Speckle noise suppression is a prerequisite for many Synthetic Aperture Radar (SAR) image processing tasks. Ultrasound images are high resolution images which would get affected by speckle noise [1]. Image quality significantly degrade by speckle noise and hence, makes it more difficult for the observer to discriminate fine detail of the images in diagnostic analyses. Speckle noise is a form of multiplicative noise, so it makes visual Interpretation difficult. Speckle noise degradation is more within bright areas rather than in dark areas of an image. Linear and nonlinear methods are available for noise removal [2]. One big advantage of linear noise removal models (Mean, Median, and Winner) is the speed, but a disadvantage of the linear models is that they are not capable to preserve edges in a good manner. There are different nonlinear filters such as Frost, Kuan, Lee etc. which are used to suppress the speckle noise. The above filtering methods would damage the image contents and also the original resolution would get reduced. Multi resolution Wavelet domain methods would overcome these problems [3].

The Discrete Wavelet Transform (DWT) has some difficulties that undetermined its application in image processing as poor directional selectivity and lack of shift invariance. Wavelet transform fails to give sparse representation along $c^{2}$ curve [4]. Wavelet effectively represent discontinuities for one dimension signal. Significant growth in the development of directional wavelets has been made in latest years. The complex wavelet transform improves directional selectivity and only requires $\mathrm{O}(\mathrm{N})$ computational cost. But, the complex wavelet transform has not been widely used in the past, because it is difficult to design complex wavelets with perfect reconstruction properties and good Filter characteristics. Another popular technique is the dual-tree complex wavelet transform (DT CWT) proposed by Kingsbury, which added perfect reconstruction to the other attractive properties of complex wavelets, six directional selectivities, including approximate shift invariance, limited redundancy and efficient $\mathrm{O}(\mathrm{N})$ computation[5]. The 2D complex wavelets are essentially constructed by using tensorproduct 1D wavelets. The directional selectivity provided by complex wavelets (six directions) is much better than that obtained by the classical DWT (three directions), but is still limited. Multi resolution curvelet transform would overcome these problems. The denoised image performances are evaluated using quality metrics like signal to noise ratio, figure of merit etc.

\section{The Curvelet Transform}

A new multi-scale transform curvelet is after 1999 which is based on wavelet transform [5]. Curvelet tronsform's structural elements include the location and parameters of dimension, and orientation parameter more, which let curvelet transform has good orientation characteristic. That's why curvelet transform is superior to wavelet in the expression of image edge, like geometry characteristic of curve and beeline, which has already obtained good research results in image denoising.

Curve-lets are initially introduced by Candes and Donoho. The idea of the Curve-let transform is first to decompose the image into sub-bands, i.e. to separate the object into a series of disjoint scales[6]. The Discrete Curvelet transform (DCT) takes as input a Cartesian grid of the form $f(n 1, n 2)$ (We work on ultrasound image so this $f$ represent the 2D ultrasound image and $n 1$ and $n 2$ are number of rows and columns of that image respectively) and the output coefficients $\mathbf{C}_{(\mathbf{j}, \mathbf{j}, \mathbf{k})}^{\mathbf{D}}$ defined by

$$
\mathbf{C}_{(\mathrm{j}, \mathrm{k}, \mathrm{l})}^{\mathrm{D}}=\Sigma_{n 1, n 2} f\left(n \mathbf{1}_{2}, n 2\right) \boldsymbol{\omega}_{\mathrm{j}, \mathrm{k}, \mathrm{l}}^{\mathrm{D}}\left(\mathbf{n}_{1}, \mathbf{n}_{2}\right)
$$

Where $\boldsymbol{\omega}_{\mathbf{j}, \mathbf{k}, \mathbf{l}}^{\mathbf{D}}\left(\mathbf{n}_{\mathbf{1}}, \mathbf{n}_{\mathbf{2}}\right)$ are digital curvelet waveforms which preserve the listed properties of the continuous curvelet. If we compare the curvelet system with the conventional Fourier and wavelet analysis, than short-time Fourier transform uses a shape-Fixed rectangle in Fourier domain, likewise conventional wavelets use shape-changing (dilated) but Fixedarea windows [6]. Same way the curvelet transform uses angled polar wedges or angled trapezoid windows in frequency domain in order to resolve also directional features.

\section{Image Denoising}

Image De-noising is used to produce good estimates of the original image from noisy observations. The recovered image should contain less noise than the observations while still keep sharp transitions (i.e edges) [7]. 
Proc. of the Intl. Conf. on Advances in Computing, Control and Networking - ACCN 2015.

Copyright (C) Institute of Research Engineers and Doctors, USA .All rights reserved.

ISBN: 978-1-63248-038-5 doi: 10.15224/ 978-1-63248-038-5-05

Image de-noising techniques vary from simple thresholding to complicate model based algorithm. However simple thresholding methods can remove most of the noise.

\section{A. Speckle Suppression Algorithm Based on Transform}

The image denoising consists of various steps as follows,

1. Apply the Forward WaveletlCurvelet transform to the noisy image.

2. Threshold the WaveletlCurvelet co-efficients to remove some insignificant Waveletlcurve-let co-efficients by using a thresholding (Hard, Soft) function in the Waveletlcurvelet domain.

3. Inverse WaveletlCurvelet transform of the thresholded co-efficients to reconstruct a function.

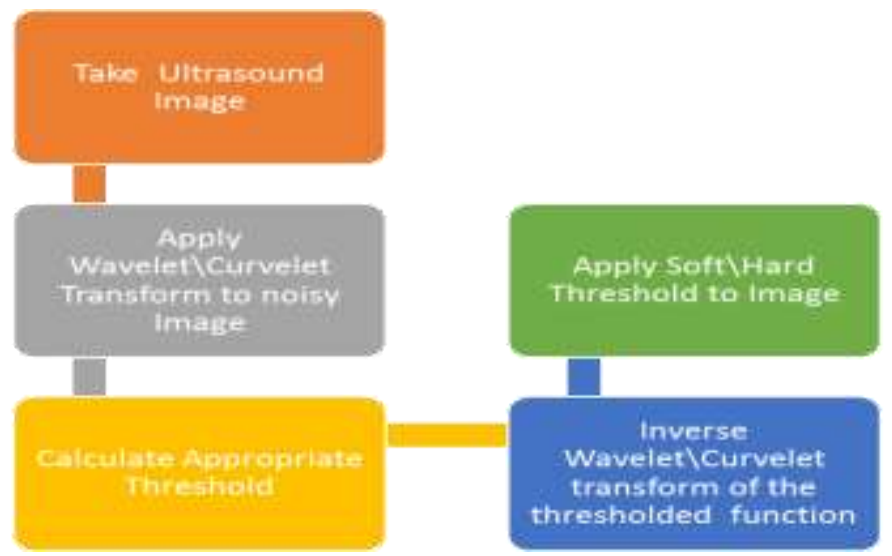

Figure 1. Speckle Denoising Algorithm

\section{B. Thresholding Function}

The term Waveletlcurvelet thresholding is defined as decomposition of the data of image into waveletlcurvelet coefficients, comparing the detailed coefficients having a given threshold value, and minimizing these coefficients close to zero to remove the effect of noise in the data. Then image is reconstructed from modified coefficients [8].

At the time of thresholding, a waveletlcurvelet coefficient is compared to the given threshold and is set to zero if its magnitude is less than the threshold otherwise, it is then retained or changed depending on the thresholding rule [9]. Thresholding distinguishes between coefficients due to noise and consisting of important image information [2]. The threshold selection is an important point of interest. It plays an important role in the removal of noise in the images because image de-noising methods most frequently produces smoothed images, by reducing the sharpness of the image. Care should be taken to save the edges of the de-noised image. Typically used methods for image noise removal include Suresh, Visu and Bayes. In this paper we use Bayes Thresholding method.

1. Soft Thresholding (shrinkage threshold)
Soft Thresholding is also called as a shrinkage threshold involves first setting to zero the elements whose absolute values are lower than the threshold and then scaling the nonzero coefficients toward zero [1].

$$
\begin{array}{ll}
\mathrm{S}_{\sigma}(\mathrm{x})= & \mathrm{x}-\sigma \quad \sigma \\
0 & |\mathrm{x}|<0 \\
\mathrm{x}+\sigma & \mathrm{x} \leq \sigma
\end{array}
$$

\section{Hard Thresholding}

where the wavelet coefficients are preserved if they are greater than the threshold, otherwise they are set to zero

$$
\begin{array}{rr}
\mathrm{S}_{\sigma}(\mathrm{x})=\mathrm{X} & |\mathrm{x}| \geq \sigma \\
0 & |\mathrm{x}|<\sigma
\end{array}
$$

Soft thresholding avoids spurious oscillations since it eliminates the discontinuity that is inherent in hard thresholding. These thresholding functions might be a good choice because large co-efficients remain nearly unaltered [1]. In partial reconstruction the image was reconstructed using the few largest co-efficients and the remaining co-efficients were set to zero.

\section{Parameters to be compared}

Following subsections describe some qualitative parameters for evaluation of quality of de-noising methods in detail.

\section{Mean Square Error (MSE)}

MSE is widely used to find the total amount of differences between the original and the de-noised image. lower and Higher MSE values indicate smaller and larger differences between the original and filtered image, respectively [10]. MSE is equal to zero for identical images. It is 255 for completely dissimilar images. It is calculated as follows:

$$
M S E=\frac{1}{M N} \sum_{i=1}^{M} \sum_{j=1}^{N}\left(X_{i, j}-X_{i, j}^{\prime}\right)^{2}
$$

Signal-to-Noise Ratio (SNR)

SNR is a common measurement to evaluate the speckle reduction in the case of multiplicative noise by computing the ratio between the original and the de-noised image. Higher SNR values show that the filtering effect is better, and filtered image quality is much higher.

\section{Peak Signal-to-Noise Ratio (PSNR)}

PSNR is measurement of the performance of the speckle noise reduction. It is a ratio between the maximum possible power of the signal and the noise image. The PSNR can be calculated as follows [10]:

$$
P S N R=10 \log _{10} \frac{\left(2^{n}-1\right)^{2}}{M S E}=10 \log _{10}\left(\frac{255^{2}}{M S E}\right)
$$

where MSE is a Mean Square Error computed between original and de-noised image. Higher PSNR values correspond 
to a better image quality. For identical images, the MSE becomes zero and the PSNR is undefined.

\section{Speckle Index Ratio (SIR)}

SI is a measure of speckle reduction in terms of average contrast of the image. If the value of SI is lower than quality of reconstructed image is good [10]. The SI is defined as follows:

$$
S I=\frac{1}{M N} \sum_{i=1}^{M} \sum_{j=1}^{N} \frac{\sigma(i, j)}{\mu(i, j)}
$$

where $\sigma$ is Standard Deviation and $\mu$ is mean value.

\section{Image Quality Index (IQI)}

It is a measure used to find the image distortion. It's mathematically defined by making the image distortion relative to the reference image as a combination of three factors: Luminance distortion, Loss of correlation and contrast distortion. The best value 1 is achieved if and only if the images are identical.

$$
Q=\frac{\sigma_{j}}{\sigma_{f} \sigma_{j}} \cdot \frac{2 \bar{f} \bar{f}}{f^{2}+\hat{f}^{2}} \cdot \frac{2 \sigma_{f} \sigma_{j}}{\sigma_{f}^{2}+\sigma_{j}^{2}}
$$

\section{Mean SSIM}

The luminance of the surface of an object being observed is the product of the reflectance and the illumination, but the object's structures in the scene are independent of the illumination [2]. To explore the structural information in an image, we wish to separate the influence of the illumination. Structural information in an image as those attributes that represent the structure of objects, independent of the average luminance and contrast. we combine the three comparisons of and name the resulting similarity measure the SSIM index In practice, one usually requires a single overall quality measure of the entire image. We use a mean SSIM (MSSIM) index to evaluate the overall image quality

$$
\operatorname{MSSIM}=\frac{1}{M} \sum \operatorname{SSIM}\left(\mathrm{X}_{\mathrm{i}}, \mathrm{Y}_{\mathrm{j}}\right)
$$

where $\mathrm{X}$ and $\mathrm{Y}$ are the reference and the distorted images, respectively; $\mathrm{Xj}$ and $\mathrm{Yj}$ are the image contents at the th local window; and $\mathrm{M}$ is the number of local windows of the image. Depending on the application, it is also possible to compute a weighted average of the different samples in the SSIM index map [2]. It has been observed that different image textures attract human fixations with varying degrees.

\section{Feature of Merit (FOM)}

Researchers have considered the problem of measuring edge detector performance. Actually, it is difficult because we don't really know what the underlying features are that we wish to detect. So, if we adopt that they are step edges corrupted by Gaussian noise, then some criteria can be set for calculating performance. Such criteria are usually the following:

- $\quad$ the probability of missing edges;
- the probability of false edges;

- the error in estimating the edge angle;

- the mean square distance of the edge estimate from the true edge; and

- the algorithm's tolerance to distorted edges and other The scientist Pratt introduce function FM for measuring quantitatively the performance of various edge detectors. The equation for measure is

$$
\mathrm{FM}=\frac{1}{\max \left(\mathrm{I}_{\mathrm{A}}, \mathrm{I}_{\mathrm{I}}\right)} \sum_{\mathrm{i}=1}^{\mathrm{I}_{\mathrm{A}}} \frac{1}{1+\mathrm{d}_{\mathrm{i}} \alpha^{2}},
$$

where $I_{I}, I_{A}, d$, and $\alpha$ are respectively the ideal edges, the detected edges, the distance between the actual and the ideal edges, and a design constant used to penalize displaced edges.

\section{Experimental Results}

Speckle noise is a multiplicative noise in nature and it is difficult to remove. It mostly affects the Ultrasound images and the image quality is degraded. We apply our wavelet\Curvelet thresholding methods on two different ultrasound images. Original Images are taken from Zydus hospital, Anand, Gujarat, India.

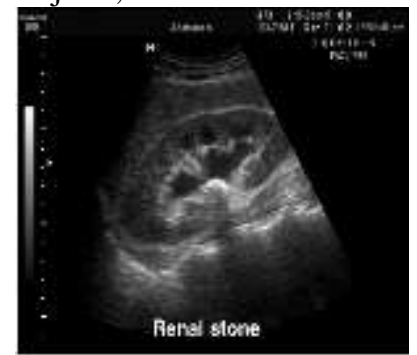

Figure 2. Original Ultrasound Image-1.

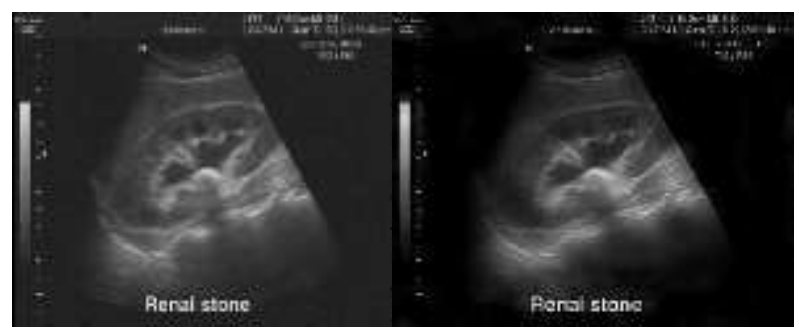

Figure 3. Hard Thresholding using Figure 4. Soft Thresholding using Curvelet Transform

Curvelet Transform

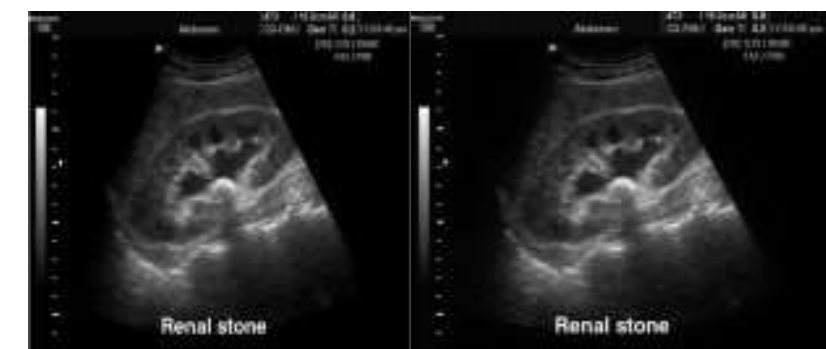

Figure 5. Hard Thresholding using Figure 6. Soft Thresholding using Wavelet Transform Wavelet Transform 
For remove the Speckle noise apply wavelet/curvelet/ transform to the noisy ultrasound image. In discrete wavelet transform the decomposition level is 2. Then WaveletlCurvelet coefficients are estimated. Then apply the appropriate threshold. The inverse waveletlCurvelet transform is applied to that estimated threshold coefficients to get the reconstructed image. Figure 2 Shows the original ultrasound image-1 which is degraded by speckle noise. Fig. 3 shows the results of Ultrasound image denoising through curvelet hard thresholding filtering. Figure 5 indicate denoised image after Wavelet hard thresholding filtering. Figure 4 and Figure 5 represent soft thresholding method using curvelet and wavelet transform simultaneously.

Practically, observing at the figures the soft method is much enhanced and yields more visually pleasant images. This is because the hard method is discontinuous and yields abrupt artifacts in the images recovered. And the second observation is for edges. Curvelet transform much preserve the edges of the ultrasound image rather than the wavelet transform.

Figure 7 is another kidney stone ultrasound image. We can easily see that because of the speckle anyone can not exactly calculate the exact size of the stone. Figure 8 to Figure 11 are the denoised version of original image as explain earlier.

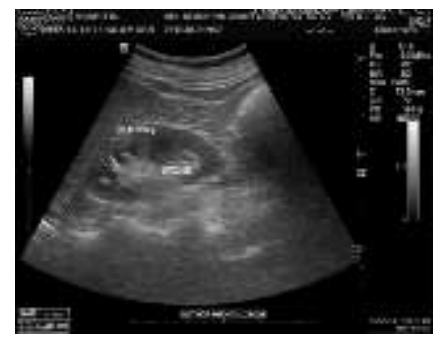

Figure 7. Original Ultrasound Image-2.

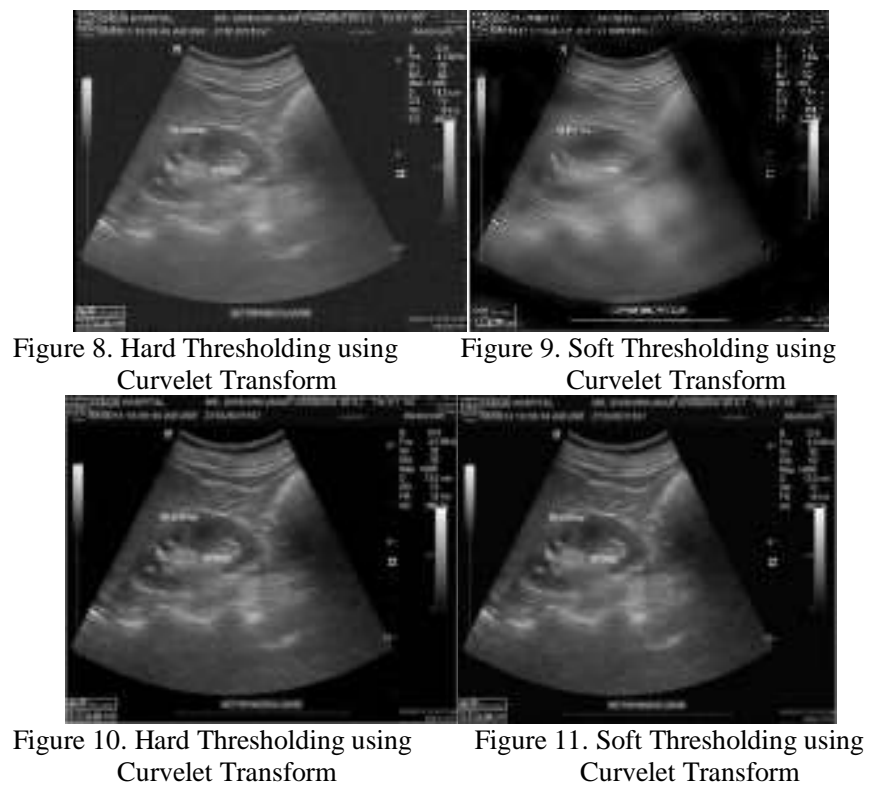

The performance of the proposed wavelet and curvelet method is evaluated using the quality metrics Measurement shown in Table 1 and Table 2 of two different ultrasound images simultaneously. After analyzing the results shown in Tables 1 and 2, we find that wavelet has more speckle noise removal power than the curvelet transform because the value of the PSNR is more in wavelet transform, and we can also see that wavelet transform remove the speckle more efficiently because the value of speckle index of wavelet transform is less compare to curvelet transform.

TABLE1 1. Comparison of Quality Metrics of Image-1

\begin{tabular}{|l|l|l|l|l|}
\hline $\begin{array}{l}\text { Quality } \\
\text { Metrics }\end{array}$ & $\begin{array}{l}\text { Curvelet } \\
\text { Hard } \\
\text { Thresholding }\end{array}$ & $\begin{array}{l}\text { Wavelet } \\
\text { Hard } \\
\text { Thresholding }\end{array}$ & $\begin{array}{l}\text { Curvelet } \\
\text { Soft } \\
\text { Thresholding }\end{array}$ & $\begin{array}{l}\text { Wavelet } \\
\text { Soft } \\
\text { Thresholdi } \\
\text { ng }\end{array}$ \\
\hline MSE & 71.65 & 48.09 & 109.75 & 103.62 \\
\hline SNR & 70.06 & 71.82 & 68.21 & 68.48 \\
\hline PSNR & 29.57 & 31.30 & 27.72 & 27.97 \\
\hline SI & $3.7 \mathrm{e}-06$ & $3.6 \mathrm{e}-06$ & $3.6 \mathrm{e}-06$ & $3.6 \mathrm{e}-06$ \\
\hline IQI & 0.0300 & 0.0320 & 0.0349 & 0.0326 \\
\hline MSSIM & 0.8020 & 0.8260 & 0.7767 & 0.5993 \\
\hline FOM & 74.86 & 71.79 & 79.63 & 78.20 \\
\hline
\end{tabular}

Experimental results shows that the value of the figure of merit (FOM) is better in curvelet transform. So, we can say that the curvelet method can better preserve the edges compare to wavelet transform.

TABLE1 2. Comparison of Quality Metrics of Image-2

\begin{tabular}{|l|l|l|l|l|}
\hline $\begin{array}{l}\text { Quality } \\
\text { Metrics }\end{array}$ & $\begin{array}{l}\text { Curvelet } \\
\text { Hard } \\
\text { Thresholding }\end{array}$ & $\begin{array}{l}\text { Wavelet } \\
\text { Hard } \\
\text { Thresholding }\end{array}$ & $\begin{array}{l}\text { Curvelet } \\
\text { Soft } \\
\text { Thresholding }\end{array}$ & $\begin{array}{l}\text { Wavelet } \\
\text { Soft } \\
\text { Thresholdi } \\
\text { ng }\end{array}$ \\
\hline MSE & 119.71 & 76.51 & 109.75 & 130.51 \\
\hline SNR & 69.21 & 71.16 & 68.21 & 68.97 \\
\hline PSNR & 27.34 & 29.29 & 26.72 & 26.97 \\
\hline SI & $2.6 \mathrm{e}-06$ & $2.7 \mathrm{e}-06$ & $2.5 \mathrm{e}-06$ & $2.5 \mathrm{e}-06$ \\
\hline IQI & 0.0218 & 0.0211 & 0.0236 & 0.0224 \\
\hline MSSIM & 0.7552 & 0.7705 & 0.6931 & 0.4451 \\
\hline FOM & 80.28 & 72.23 & 81.07 & 80.75 \\
\hline
\end{tabular}

\section{Conclusion}

Ultrasound image contain speckle noise and the multiplicative nature of speckle noise is difficult to remove. The curvelet transform method has performed better in reducing the speckle noise while preserving edge information. Also, it likely that the wavelet method is more effective in reducing speckle noise while the curvelet method can better preserve the edges. The performance of both Speckle noise reduction method is evaluated by quality metrics measurement like PSNR, MSE, SNR, SI, MSSSIM, IQI and FOM. 
Proc. of the Intl. Conf. on Advances in Computing, Control and Networking - ACCN 2015.

Copyright (C) Institute of Research Engineers and Doctors, USA .All rights reserved.

ISBN: 978-1-63248-038-5 doi: 10.15224/ 978-1-63248-038-5-05

\section{References}

[1] Gregorio Andria, Anna M. L. Lanzolla, "A Suitable Threshold for Speckle Reduction in Ultrasound Images," IEEE Transactions on Instrumentation and Measurement, VOL. 62, NO. 8, August 2013

[2] Nandhini G, Saraswathy C.," SAR image despeckling using wavelet and curvelet transform," International Journal of P2P Network Trends and Technology (IJPTT) - Volume3 Issue4- May 2013

[3] Mohamed Ali HAMDI, "A Comparative Study in Wavelets, Curvelets and Contourlets as Denoising Biomedical Images," I.J. Image, Graphics and Signal Processing, 2012, 1, 44-50.

[4] JIANG Tao ZHAO Xin. Nicole, "Research and Application of Image Denoising Method Based on Curvelet Transform," The International Archives of the Photogrammetry, Remote Sensing and Spatial Information Sciences. Vol. XXXVII. Part B2. Beijing 2008.

[5] A. Anilet Bala1, Chiranjeeb Hati and CH Punith, "Image Denoising Method Using curvelet Transform and Wiener Filter International Journal of Advanced Research in Electrical, Electronics and Instrumentation Engineering (An ISO 3297: 2007 Certified Organization) Vol. 3, Issue 1, January 2014.

[6] Anil A. Patil, Jyoti Singhai, "Image Denoising using Curvelet Transform:an approch for edge prevention," J SCI IND RESVol 69 January 2010

[7] Miss Monika shukla1, Dr.Soni changlani, "A Comparative Study of Wavelet and Curvelet Transform for Image Denoising," IOSR Journal of Electronics and Communication Engineering (IOSR-JECE) e-ISSN: 2278-2834,p- ISSN: 2278-8735. Volume 7, Issue 4 (Sep. - Oct. 2013), PP 63-68.

[8] Mohammed Tarek GadAllah, Samir Mohammed Badawy."A Wavelet Based Approch for Ultrasound Restoration" (IJACSA) International Journal of Advanced Computer Science and Applications, Vol. 5, No. 7, 2014"

[9] D. Mary sugantharathnam, Dr. D. Manimegalai," The Curvelet Approach for Denoising in various Imaging Modalities using Different Shrinkage Rules" International Journal of Computer Applications (0975 - 8887) Volume 29- No.7, September 2011.

[10] Savaliya Nirali H., Shah Manasi J.,Sheth Dhrumil H., Raviya Kapil S," Analysis of Renal Calculi in Ultrasound Image Using Matlab," Journal of Information, Knowledge and Research in Electronics and Communication Engineering.

[11] Sheth Dhrumil, Saurin Shah," Ultrasound Image Segmentation Techniques for Renal Calculi - A Review," European Journal of Academic Essays 1(10): 51-55, 2014,ISSN (online): 2183-1904

[12] J.C.Acharya, K.S.Raviya, "Objective Asseement of different segmentation algorithm for under water images", $4^{\text {th }}$ IEEE international conference on computing, communication and networking technologiesIEEE-31661-2013

[13] K. Shahnazi and M. Fox, "Speckle Reduction in Real Time Ultrasound Imaging", () 1994 IEEE

[14] H. Lazrag, M. Ali H., and M. Saber N., "Despeckling of Intravascular Ultrasound Images using Curvelet Transform", SETIT, March 2012.

[15] F. Y. Rizi, H. A. Noubari, and S. K. Setarehdan, "Wavelet-Based Ultrasound Image Denoising: Performance Analysis and Comparison", 33rd Conf. IEEE-EMBS, Boston, (2011 IEEE

[16] J. Starck, E. J. Candès, and D. L. Donoho, "The Curvelet Transform for Image Denoising", IEEE Transactions on Image Processing, Vol. 11, No. 6, June 2002, pp. 670-684, (C) 2002 IEEE

[17] M. Elhabiby ... et al, "Second Generation Curvelet Transforms Vs Wavelet transforms and Canny Edge Detector for Edge Detection from WorldView-2 data", IJCSES, Vol.3, No.4, Aug. 2012

[18] David L. Donoho and Mark R. Duncan, "Digital Curvelet Transform: Strategy, Implementation and Experiments", Technical Report No. 2000-12, Department of Statistics, Stanford University, March, 2000. Available: http://statweb.stanford.edu/ ckirby/techreports/GEN/2000/2000-12.pdf

[19] K. Ding, "Wavelets, Curvelets and Wave Atoms for Image Denoising", 3rd International CISP2010, (2)2010 IEEE
[20] Effectiveness of Contourlet vs Wavelet Transform on Medical Image Compression: a Comparative Study Negar Riazifar, and Mehran Yazdi World Academy of Science, Engineering and Technology 492009.

[21] E. J. Candès, L. Demanet, D. L. Donoho, and L. Ying, "Fast discrete curvelet transforms," Multiscale Model. Simul., vol. 5, no. 3, pp. 861899, Jan. 2006

[22] E. J. Candès and D. L. Donoho, "Curvelets-A surprisingly effective nonadaptive representation for objects with edges," in Curves and Surface Fitting: Saint-Malo 1999, A. Cohen, C. Rabut, and L. Schumaker, Eds. Nashville, TN: Vanderbilt Univ. Press, 2000, pp. 105120.

About Authors:

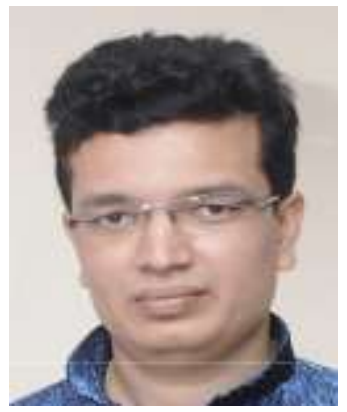

Mr. Dhrumil H. Sheth has completed his bachlor and masters degree in the Electronics \& Communication Engineering from Gujarat university, Ahmedabad, gujarat, india and he is currenly pursuing his $\mathrm{Ph}$. D. in the same discipline from C.U.Shah University Wadhawan, Gujarat, India. His Area of reaserch is to medical Image Processing. He is currently working as an assistant professor in the department of electronics \& communication engineering C.U.Shah College of Engineering and Technology Wadhawan, gujarat, india.

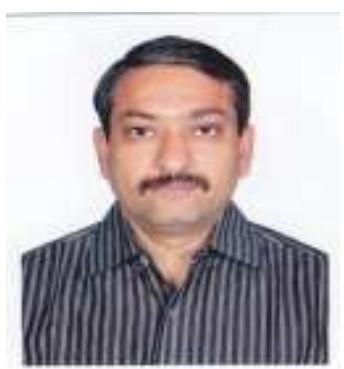

Dr Saurin R. Shah is Professor and Principal, Silver Oak College of Engineering and Technology, Ahmedabad, Gujarat. He has published a number of research papers in journals, both national and international. $\mathrm{He}$ obtained his M.Tech from I.I.T. Madras and Ph.D from Nirma University, Ahmedabad, Gujarat. He has been Involved in Teaching and research in areas of Image Processing, signal processing, analog and digital system design. 